\title{
Evolution of online discussion forum richness according to channel expansion theory: A longitudinal panel data analysis
}

\begin{abstract}
:
The selection and use of communication media has been the centre of attention for a great number of researchers in the area of organizational communication. The channel expansion theory combines elements of the main theories in this area; however, these investigations have a static cross-sectional design rather than a longitudinal analysis. With the objective of filling this gap, we research how the perception of media richness varies over time with a longitudinal study. The results suggest that the perception of richness of a channel by different individuals is different but that those variations due to a change in acquired experiences, are influenced in a similar and significant way by all individuals over time.
\end{abstract}

\section{Keywords:}

Channel expansion theory; panel data; online discussion forum; media richness

\section{Introduction}

Existing literature examines the importance of organizational communication in the operational efficiency of an organization and consequently in the attainment of its objectives (Dahle, 1954). For this reason, the selection and use of communication media has, for decades, been the center of attention for a great number of scholars in the area of organizational communication. 
Nevertheless, an agreed theory does not exist on the selection and the use of such media, but rather the opposite: a great number of interrelated theories have been developed on the causes that determine these decisions.

The main theories on the selection and use of communication media are, media richness theory (Daft \& Lengel, 1984, 1986), social influence theory (Schmitz \& Fulk, 1991), media symbolism theory (Trevino, Lengel, \& Daft, 1987), situational factors theory (RE Rice, 1992; Trevino et al., 1987), critical mass theory (Markus, 1987), communication genre theory (Yates \& Orlikowski, 1992), channel expansion theory (Carlson \& Zmud, 1999), social presence theory (Short, Williams, \& Christie, 1976), and media synchrony theory (Dennis \& Valacich, 1999). These theories can be represented as a continuum (Vishwanath, 2006), placing those theories based on rational decision making at one extreme and other theories emphasizing social aspects at the other. Those theories based on rational decision making concentrate on the characteristics of the communication media (for example, its bandwidth) in order to determine its capacity to transmit meaning and intention to those receiving the messages; on the other hand, theories that emphasize social aspects concentrate on how the surroundings (for example, work partners and organizational culture) affect the choice and use of the communication media. Despite the great number of existing theories, media richness theory and social influence theory have been the most predominant theories in the literature when examining how and why members of an organization select a particular media to communicate with other people or institutions (Te'eni, Sagie, Schwartz, Zaidman, \& Amichai-Hamburger, 2001). These theories have been the main departure point for the rest of existing theories in the literature.

The channel expansion theory combines elements of media richness theory with certain characteristics of influence and social presence theories. Initially, Carlson and Zmud (1999) tested this theory in only one communication media: traditional electronic mail. The results agreed with the majority of 
proposals made by channel expansion theory. An analysis of the existing literature shows that there have been no further verifications of this theory for several years. Recently, several investigations have been published (for example, D'Urso and Rains (2008) and Timmerman and Madhavapeddi (2008)) in which channel expansion theory has been studied directly in several communication media. However, these investigations have a static crosssectional design (individuals in just an specific one moment in time), a snapshot of channel expansion theory rather than a longitudinal analysis which allows for the evolution of media richness to be observed over time.

Using investigations undertaken on the channel expansion theory as a departure point and with the aim of taking a further step towards its understanding, we studied how the perception of media richness varies over time. With this objective, we developed a longitudinal investigation on a distance research business course where the students used several on-line discussion forums as their main communication tool with their lecturers and classmates. We have found evidence that the individual's acquired experiences (the experience with the channel and with the communication partner) are a significant antecedent of changes over time of the perception of the richness of a channel. Also, the results obtained suggest the existence of other factors in individuals' perceived richness related to the time factor. Finally, this investigation suggests several theoretical and practical implications to improve management efficiency in organizations, as well as some limitations and proposals for future research.

\section{Background}

\subsection{Media Richness and Social Influence}

Media richness theory has become one of the most studied and referenced theories in the area of investigation on selection and use of communication media in organizations. Media richness theory describes the rational process 
that managers and workers follow when communicating. According to this theory, the choice of communication media is defined by the relation between the objective capacity of the communication media and the communication tasks' requirements. The capacity or richness of a medium is defined as the capacity of the medium to develop a common and shared meaning between the broadcaster and the receiver of the message, on which the notion of the richness of information is based (Daft \& Lengel, 1984). This media richness is determined by four intrinsic characteristics of the communication media; the ability to offer fast feedback (which can be either concurrent or sequential), send multiple cues through multiple communication channels (for example, an emotional tone, attitude or formality), allow for different types of language (for example, text, graphics and tables), offer the possibility of personalizing messages based on the receiver and the context in which the communication takes place.

The evaluation of media richness is a subject which has been dealt with on several occasions, where work by Ferry, Kydd, and Sawyer (2001) particularly stands out. According to the original theory of media richness, channels can be distributed along a continuum based on their level of richness (Rice, 1992). According to this continuum, the communication medium with the greatest richness is face-to-face communication, followed by telephone communication. Memos and numerical documents lie at the other end of the continuum, as the media with a lower level of richness According to this sort, oral communication usually has a greater degree of richness than written communication. Also, synchronous communication media usually have greater richness than asynchronous ones. Initially, media richness theory has been examined in traditional communication media (for example, face-to-face, telephone, e-mail and letters) with results that supported the theory's proposals; however, few investigations have studied or supported the theory with more modern communication media (for example, videoconferencing, instant messaging, and voicemail). 
Recently, Kishi (2008) analyzed a great number of traditional and modern communication media together (face-to-face, meetings, teleconferences, videophone, free discussions, cordless phone, telephone, e-conferences, letter, memo, email, fax, and pager) according to media richness theory. The results suggest the use of communication media by managers is positively related to the probability of using traditional communication media with a high degree of richness, if it is considered that the organization's external environment is not analyzable. Furthermore, the results suggest that the use of communication media (traditional and modern) by managers is not related to their degree of richness if there is a culture to reveal and show personal and professional. messages to other people of the organization.

From a completely different perspective, several investigations suggest that the selection and use of communication media depends on non-objective characteristics, more specifically, of the subjective perceptions of the users or work groups that participate in the communication (Fulk \& Boyd, 1991). These perceptions are defined by two determinants: the differences that exist between individuals (inherent aspects of the user and their experience with the media) and social information (Fulk, 1993). According to Timmerman (2002), the comments and the use of communication media by relatives, friends, and partners are key elements in the selection and use of a these media. For example, the use of a social net (such as Facebook or Twitter) depends primary on the use of relatives and friends, more than the features of the social net.

Whereas media richness theory focuses on the objective characteristics of communication media and the type of activity, social influence theory makes reference to the society and people features that influence the selection and use of communication media.

\subsection{Channel Expansion Theory}


From a combination of the main concepts of media richness and social influence theories, Carlson and Zmud (1999) introduces the channel expansion theory. This theory also draws partially on other theories such as situational factor theory (Trevino et al., 1987) and social presence theory (Short et al., 1976). Channel expansion theory maintains that media richness is a crucial element in the selection and use of media; however, the conception that media richness is constant in all situations and for all individuals in an organization disappears.

Channel expansion theory states that knowledge-building experiences of the members of an organization influence the perception of media richness. The experience with a communication channel has been evaluated in terms of time length (Fulk, 1993) or the number of times that a channel has been used (Rice \& Love, 1987); however, channel expansion theory suggests that knowledgebuilding experiences have a greater effect on selection and use of communication media than these variables. In short, this theory considers that the selection and use of communication media depends on the perception of each medium by the members of an organization, based on their existing knowledge base. As D'Urso and Rains (2008) states, the idea that the experience of the members of an organization is associated to the interpretations of a communication medium is consistent with existing literature on the relational development of communication via computers (for example, Walther $(1992,1995))$.

Channel expansion theory identifies certain knowledge-building experiences that modify the way in which an individual in an organization develops their perception of media richness. Carlson and Zmud (1999) identified four knowledge-building experiences: experience with the channel, experience with the subject, experience with the communication partner and experience with the organizational context. 
The literature suggests that experience with a communication channel by an individual will allow him to learn the characteristics, the options, the uses and the limitations of the channel, allowing him to use the communication channel more efficiently, adapting its use to the characteristics of the task required. The experience with a communication channel will also increase its perceived richness by the individual.

In a similar way to how individuals acquire experience with a channel through communication, these individuals also acquire experience with their communication partners. These experiences consist of interactions and mutual learning which take place between the two or more individuals, such as language patterns and expectations in the construction of messages. Such mutual learning allows for the use of a richer language in communications and to codify messages for the receiver, allowing for relevant cues specifically for him or her. For example, two individuals can use a shared past experience to deal with a present subject or to use phrases with connotations only they understand.

Timmerman and Madhavapeddi (2008) proposes that knowledge-building experience of the topic can only be developed after increasing knowledgebuilding experience of the communication media and the partner of the communication. The knowledge of a topic allows individuals to use specialized, familiar jargon and make communications between them easier. Consequently, it seems that there is no need to construct a topic-based shared knowledge base to increase the perception of media richness. Finally, the use of symbols and shared cultural references in an organization allows for a richer communication through the media due to the increase in the sophistication of the organization's knowledge base.

Carlson and Zmud (1999) also proposed that the perception of media richness by an individual, and consequently of the selection and use a communication media, depends directly on the social influence in the organization. In a certain 
way, those knowledge-building experiences of organizational context and of the communication partners and social influence are closely related elements, because the organizational context and the kind of members in an organization determine the degree and type of social influence that exists in an organization. As Timmerman and Madhavapeddi (2008: 20) sets out "the social influences in an organization on the use of media should also establish another type of knowledge base, and as a result, should be associated to the perceptions of media richness".

Until recently, a reduced number of investigations (Carlson \& Zmud, 1999; D'Urso \& Rains, 2008; Timmerman \& Madhavapeddi, 2008) have focused their attention on testing the premises of channel expansion theory through cross-sectional studies. However, there are some evidences in the literature supporting that knowledge-building experiences can expand the perception of the richness of a medium, allowing their users to achieve a level of task complexity above the expectations of media richness theory.

The study by Carlson and Zmud (1999) brought evidence of the relationship between knowledge-building experiences and perceptions in the richness of electronic mail. The results of this investigation proposed that knowledgebuilding experiences had a greater impact on the variability in the richness perception of electronic mail than its frequency of use. More specifically, the study suggested that the hypotheses on the relations between the richness of a medium and the knowledge-building experiences of the medium and the communication partner were upheld, that the hypothesis on the relation between the richness of the medium and knowledge-building experience of the organizational context was partially upheld, and that no direct relation existed between media richness and knowledge-building experience of the topic or social influence.

The investigation by Timmerman and Madhavapeddi (2008) replicated the original study by Carlson and Zmud (1999) with electronic mail, adding two 
new communication media: face to face and the telephone, using a sample of 529 surveys. The results obtained from this investigation suggested that perceptions of a medium's richness are positively related to knowledgebuilding experiences with the medium, the communication partner, topic, and social influences. These relationships are fairly consistent between face-toface, telephone and email. Differences in richness are attributable to differences between the media, expansion variables appear to be selectively related to richness dimensions, and these relationships vary between media.

The main limitation of that work was the choice of traditional media instead of more up-to-date and less common communication media in organizations such as videoconferencing or instantaneous messaging. However, the results confirm, to a large extent, the results obtained by Carlson and Zmud (1999) for this type of communication media. Finally, D'Urso and Rains (2008) tested channel expansion theory with new communication media (instant messaging) and traditional (face to face, telephone, electronic mail) with a survey to 269 informants. The investigation results provided additional support to channel expansion theory indicating that knowledge-building experiences of the channel, with the communication partner, with the topic as well as social influence, were predictors for the perception of media richness. The main limitation of the three investigations about existing channel expansion theory (Carlson \& Zmud, 1999; D'Urso \& Rains, 2008; Timmerman \& Madhavapeddi, 2008) is the static cross-sectional design, a snapshot of the effects of channel expansion theory more than a longitudinal analysis, not allowing for the study of the evolution of the perception of media richness over time.

We therefore ask ourselves the following question for investigation:

- RQ: How does the perception of richness in a communication media evolve over time, and how are existing relations between the 
knowledge-building experiences and the perception of media richness affected?

\section{Method}

With the aim of responding to the research question, the data used had to be longitudinal. It is important to emphasize that the majority of empirical investigations on media richness are based only on cross-sectional data (individuals in a specific time), which therefore do not permit the detection of time inferences (Hitt, Gimeno, \& Hoskisson, 1998). On the other hand, longitudinal or panel data allow investigators to control the initial values of the dependent variable and to recognize temporary delay effects (Rothaermel, Hitt, \& Jobe, 2006). Panel data also allow the investigators to work with greater samples, and therefore increase the statistical power and reduce the threat of a multi-collinear relationship between the independent variables, thus allowing for an improvement in statistical estimation efficiency (Boyd, Gove, \& Hitt, 2005).

\subsection{Sample and procedures}

We developed a multivariate study incorporating an unusual communication medium for the users at four different moments in time. More specifically, the investigation reported in this paper is part of a longitudinal study (consisting of four data collection points, $t 0, t 1, t 2$ and $t 3$ ) where progress in the perception of media richness and the different determinants that have been exposed in the literature revision are evaluated (Figure 1). 
A distance postgraduate business course from a large European public university was the main source of informants for this investigation. The course was an obligatory course carrying 4.5 ECTS(European Credit Transfer System) credits, the equivalent to approximately 112 working hours for the student and which took place during the autumn semester 2008. The course had 120 registered students; however, only 95 students decided to volunteer to participate in the investigation. They were assured that their individual responses would remain confidential and that the investigators would use a summary drawn from their answers. Once the questionnaires were obtained, we eliminated all those situations in which the questionnaires were not correctly completed, discarding answers obtained from 4 students.

The final sample consisted of 91 students (25.27\% women and $74.73 \%$ men) with an average age of 29.70 years and a standard deviation of 6.20. The data were collected at four different moments during the course: at the presentation of the course, twice during the development of the course, and the day after the final evaluation test. The data were portrayed as cross-sectional and longitudinal at the same time, represented as a dataset panel. Data collection was carried through an online survey in which students were asked to identify themselves using the last 5 digits of their student ID card in order to be able to follow their development during the course. Finally, 364 (= 91 students x 4 moments) surveys were completed correctly in this investigation.

Due to the distance nature of the course, the main working and communication tool between students and lecturers was the educational platform Moodle ${ }^{1}$. Ravenscroft and Matheson (2001) suggested that online discussion forums are tools capable of supporting the educational dialogue necessary on a remote

\footnotetext{
${ }^{1}$ Moodle is a free and open source e-learning software platform, also known as a Course Management System, created by Martin Dougiamas and designed to help educators create online courses with opportunities for rich interaction (Moodle, 2009).
} 
course and to offer the level of feedback that students require. On the basis of these comments, the course lecturers introduced an online discussion forum on the educational platform, with the objective of offering a place where students and lecturers could ask, respond and discuss any topic related to the course (whether it be related to the workings of the course, or its contents, or any other topic). Lecturers included the participation of online discussion forums as a small part of the final grade. So, it can be considered that lecturers controlled students through their participation in the discussion forum. Moreover, the opinion of the rest of classmates about each student's input to the forum could be considered a kind of social supervision. Just 5.49\% of the students who participated in the investigation had used an online discussion forum previously, which offered an excellent opportunity to evaluate how the perception media richness progressed from the outset.

Level of supervision of the media has recently become one important element. According to Barry and Fulmer (2004), the degree to which other people can retrieve and read messages from an individual can affect their use. In this sense, the fact that on-line discussion forum messages could be accessed by everyone, both by teachers and by students, at any time could affect the use of the medium. For example, a comment not suitable, a stupid question, or even a lack of spelling by a student could change the opinion of classmates about him/her. This phenomenon could influence students when using the online discussion forum. On the other hand, lecturers included the participation of on-line discussion forums as a small part of the final grade. So participation in discussion forums by students could also be influenced by this kind of oversight.

\subsection{Instrumentation}


The measurements used in this investigation include original items from the investigation by Carlson and Zmud (1999) and several complementary items based on results and notable contributions from more recent investigations. The complete survey items, which were rated on a 5-point scale ( $1=$ strongly disagree, 5 = strongly agree), are provided in the Appendix. The descriptive statistics and correlations between the main variables are summarized in table 1.

\section{INSERT TABLE 1 ABOUT HERE}

Perceived media richness: The richness of a communication media is evaluated through two complementary measures. First, we included the four original items proposed by Carlson and Zmud (1999), which refer to each one of the four dimensions of the media richness construct (feedback, multiple cues, natural language, and personal focus). These items, as well their adaptations, have been the most commonly used over the last decade when evaluating the construct. Secondly, we have also included the adaptation by Hasty, Massey, and Brown (2006) of three items proposed originally by Dennis and Kinney (1998). These items were designed from the conceptual definition of the media richness construct by Daft and Lengel (1986).

Predictors of channel expansion: According to the original theory of channel expansion (Carlson \& Zmud, 1999), four kinds of experiences exist which are particularly relevant: experience with the channel, experience with the subject, experience with the communication partner, and experience of the organizational context. However, the students' organizational context experience was quite high and was not significantly different between them, so it was decided not to include it as a predictor in the investigation. The final 
survey included all the items of the measurements of experience based on knowledge from the study by Hasty et al. (2006), developed from the original measurements by Carlson and Zmud (1999). These variables include six items to evaluate channel experience, three items to evaluate experience with the topic, and six items to evaluate the experience with the communication partners. These last items had to be adapted to take into consideration both communication between students and between students and lecturers.

Supervision level: Barry and Fulmer (2004) suggested the supervision level of a communication media could influence its richness perception. The supervision level refers to the capacity of explicit supervision inherent to the media as well as to any other well-known, suspected, or anticipated type of supervision due to the technological characteristics of the media. Due to the type of activity studied in this paper, only the first type of supervision has been considered using items developed by us from their conceptual definition.

Because the main language of the objective population was different from the one in the original scales, the questionnaire was translated to Spanish. In order to assure a correct translation of the scales, we followed a backwards translation procedure (Nunnally \& Bernstein, 1994). In addition, three cognitive interviews were undertaken with the aim of assuring a correct and faithful interpretation of the questionnaire items, since this technique allows for the understanding of how those in the survey perceive and interpret the questions, and to identify potential problems that could arise from their use (Drennan, 2003). In addition, the cognitive interviews allowed us to interpret which items were beyond the theoretical framework of the constructs that were to be analyzed (Collins, 2003). By means of verbal probing and thinking aloud (Drennan, 2003; Williamson, Ranyard, \& Cuthbert, 2000) participants were asked to verbalize their interpretation of items and to paraphrase and/or comment on the wording of items in an effort to identify ambiguous or poorly worded questions. 


\section{Results and discussion}

Panel data analysis has become a very popular technique in the analysis of longitudinal data in social and behavioral sciences. This technique allows for the independent analysis of a set of individuals over the while at the same time being undertaking a cross sectional study and longitudinal one. In this particular study, we evaluate the media richness and a set of knowledge experiences of several students (cross sectional part) in different moments of a course (longitudinal part). From panel data analysis, we could test if the intercept and the coefficients of independent variables vary across the time. The general specification of the regression model of panel data in this investigation is the following:

$P M R_{i t}=\alpha_{i t}+\beta_{i t} \cdot C_{h a E x p}+\beta_{2 i t} \cdot \operatorname{TopExp}_{i t}+\beta_{3 i t} \cdot \operatorname{PartExp}_{i t}+\beta_{4 i t} \cdot \operatorname{Suver}_{i t}+\mathrm{e}_{i t}$ (1)

where $i$ represents the individuals in the study (cross section), $t$ makes reference to the temporary dimension (longitudinal section), $\alpha_{i t}$ represents the model intercepts, $\boldsymbol{\beta}_{\text {Kit }}$ are the coefficients of the model's independent

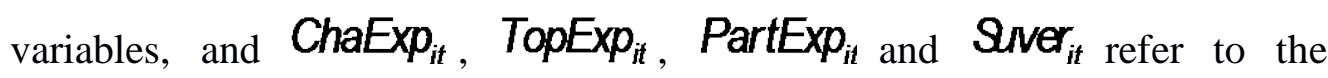
dependent variables on experience with the channel, experience with the subject, experience with the communication partner, and the supervision level respectively. Data panel can be analyzed through models with constant coefficients, models with fixed effects, and models with random effects. Among these types of models are the dynamic panel models, robust models and covariance structure models. Using model (1) we develop several analytical model types different to panel data. These new models will be based on certain supposed precedents from the literature, allowing for a better adaptation of the model to the situation analyzed in this paper. The first model analyzed is the constant coefficient model, where both the intercepts and the slopes are constant. In this model it is considered that there are neither 
significant differences between the individuals in the study nor significant differences in temporary effects. This model is commonly known as the pooled regression model, and its specification in this study is the following:

$P M R_{\text {if }}=\alpha+\beta_{1} \cdot \mathrm{ChaExp}_{\text {it }}+\beta_{2} \cdot \operatorname{TopExp}_{\text {it }}+\beta_{3} \cdot \mathrm{PartExp}_{\text {it }}+\beta_{4} \cdot \mathrm{Smer}_{\text {it }}+\mathrm{e}_{\mathrm{it}}(2)$

From the pooled regression model, two models have been proposed. Model A only considers the traditional explanatory variables proposed by Carlson and Zmud (1999): experience with the channel, experience with the subject, and experience with the communication partner. The results show that experience with the channel and experience with the communication partner are significant deciding factors in the perception of a communication media's richness $(\mathrm{p}<0.001)$. However, experience with the topic is not significant $(p=0.139)$. These results agree with those originally obtained by Carlson and Zmud (1999) and later by Timmerman and Madhavapeddi (2008), so this study provides new and convincing evidence that these two explanatory variables are essential in explaining the perceived richness of a channel. In addition, the results of this investigation extend the sample of communication channels analyzed (to include the on-line discussion forum) from the point of view of channel expansion theory.

Model B includes the explicit variable of channel supervision proposed by Barry and Fulmer (2004). The results show that the coefficient of this variable in the model is not significant $(\mathrm{p}=0.197)$ and does not improve the model's explanatory power of perceived richness of a communication channel. It is important to emphasize the use given to the communication media when analyzing this result. In this investigation an on-line discussion forum was used as a tool to resolve doubts between students and between students and lecturers. These students have been working together in many other courses, 
so their opinions about the rest of classmates were well defined. With students who have just met or younger students, the results could have been different.

On the other hand, the weight of this activity was low, so many students could have considered that the danger of the supervision on the final grade was not so important for not participating in a active way.

It is possible that the supervision level is significant when the use of communication channel is different, for example in negotiation processes (Lewicki, Barry, Saunders, \& Minton, 2003).

\section{INSERT TABLE 2 ABOUT HERE}

Model C considers that the explicit variables affect individuals equally (cross section) and that the variables are different due to their differentiating characteristics, measured by means of an intercept. This type of model in panel data analysis is known as a fixed effects model. For this, the intercepts $i$ are associated with dummy variables with specific coefficients for each temporary unit (Q2 and Q3), which must be estimated. For this particular situation, the regression model specification is the following:

$P M R_{i t}=\alpha_{1}+\alpha_{2} \cdot Q_{2}+\alpha_{3} \cdot Q_{3}+\beta_{1} \cdot C_{h a x p}+\beta_{2} \cdot \operatorname{TopExp}_{i t}+\beta_{3} \cdot \operatorname{PartExp}_{i t}+\beta_{4} \cdot \operatorname{Siver}_{i t}+e_{i t}$ (3)

where Q2 and Q3 are zero in the first period, Q2 takes the value of one in the second period while Q3 stays at zero, and Q3 takes the value of one in the third period while Q2 again equals zero. The results show that the explicit variables (channel experience and experience with the communication partner) have constant slopes for all individuals but the intercepts vary significantly 
( $<<0.001, y p=0.005$ respectively) according to the time. These results suggest that different individuals showed different overall levels of perceived media richness but that the variations can be attributable to a change in acquired experiences (channel experience, and experience with the communication partner), which influence in a similar and significant way the individuals over time.

However, this does not explain why certain individuals have a richness perception of a communication channel higher (or lower) than other individuals with similar characteristics. Also, these experiences do not completely explain the increase in richness perceived by the individuals over the periods analyzed. These differences should be analyzed in more detail in future investigations, taking into account the time factor as the results obtained suggest.

Finally, another fixed effects model was tried where the intercepts and the slopes vary according to time. In order to formulate this regression model, we have had to include not only dummy variables, but also their interactions with the explicit variables of the model. The result is the following regression model:

$$
P M R_{j i t}=\alpha_{1}+\sum_{j=2}^{3} \alpha_{j} \cdot Q_{j}+\sum_{k=1}^{4} \beta_{k} \cdot X_{k i t}+\sum_{j=2}^{3} \sum_{k=1}^{4} \gamma_{j k} \cdot Q_{j} \cdot X_{k i t}+e_{j t}
$$

where $X_{k}$ represents each one of the four explicit variables of the previous models (channel experience, topic experience, experience with the communication partner, and channel supervision). The results obtained show that all the multiplicative terms are not significant ( $\mathrm{p}>0.05)$. Because of this we can maintain that the explicit variables do not vary according to time, and suggest model $\mathrm{C}$ as the one that adapts more easily to situation under study.

\section{Conclusions}


These results have important implications for lecturers and/or managers during organizational communication. The original works on media richness (Daft \& Lengel, 1984, 1986), as well as later investigations on channel expansion theory (e.g. Carlson and Zmud (1999), D’Urso and Rains (2008), and Timmerman and Madhavapeddi (2008)), provide some prescriptive rule on the use of communication media in organizations. Now, our investigation provides additional support to the previous results on channel expansion theory by Carlson and Zmud (1999). In addition, the results suggest the need to continue work in order to reach an understanding of the elements included in the choice of new media and communication technologies, considering the effect of time as an essential element in the study. The understanding of the influence of knowledge-building experiences on the temporal evolution of media richness perception is a key element in the management of organizational communication.

The results of the investigation show that the richness of a communication media is constructed socially and is related mainly to experience with the media and with the communication partner. This result suggests that employee training in the use of communication media, as well as activities that establish constant communication between the members of an organization can increase the efficiency of organizational communications. These implications are supported from two points of view. On the one hand, critical mass theory (Markus, 1987) already suggests the need for large (critical) number of users to develop the knowledge-building experiences proposed by channel expansion theory. On the other hand, the development of knowledge-building experiences needs time for their correct assimilation by members of the organization. For this reason, the results show the importance of the time factor in the increase or decrease in the perception and usefulness of a communication media. 
In the same way as the investigation by D'Urso and Rains (2008), the results suggest the importance of general acceptance in the use of new communication media (e.g instant messaging) by the members of an organization, since the usefulness and richness perception is socially created by them, and must be created from the development of knowledge-building experiences rather than only their use.

Some investigations had suggested (e.g. Timmerman and Madhavapeddi (2008)) that the relation between experiences based on knowledge and perceived media richness could vary over time. This suggestion is not supported by our results, since the effects in the variability of knowledgebuilding experiences on the perceived media richness are constant over time; however, the time factor also has a significant effect on the richness perception, as in the fixed effects model where the intercepts vary according to time but are fixed according to the individuals (Model $\mathrm{C}$ in table 2). As the majority of investigations have verified, the simple repetition in the use of a communication media is not a factor which explains the variability of the perceived richness, which is why we think that this effect could be measured by other knowledge-building experiences that are not presently explained in the literature and which should be analyzed by an inductive investigation rather than a cross-sectional or longitudinal/quantitative investigation.

\subsection{Limitations and future investigations}

This study goes beyond previous investigations due to its longitudinal design rather than the classic cross-sectional research design, thus allowing to offer a more realistic view of the evolution of perceived richness over time. However, the assessment of only one communication medium does not allow for direct extrapolation of the results obtained to other communication media. Although the sample is fairly heterogeneous in several aspects (age, sex, work, etc.), in others it is not (level of studies). Also, all the informants belonged to a same experiment thus producing interference between the participants in the 
experiment. For this reason, new longitudinal studies are proposed following the design of this investigation, allowing in that way to compare and extrapolate the results obtained to other communication media.

A great number of areas for future investigations in relation to channel expansion theory exist. The main investigations undertaken on channel expansion theory analyze the variability of the perceived richness of one or several communication media by the members of an organization. However, there are still four important lines in which to investigate. In the first place, making reference to the identification and understanding of new determinants which affect perceived richness of the workers of an organization. As shown by the results of this investigation, only $45.8 \%$ of variability is explained by the determinants proposed by channel expansion theory.

The second line of investigation refers to the comparison, in detail, of the results obtained from new communication channels (e.g instant messaging, video stream forums and podcasting), new communication devices (e.g. iPhone, Blackberry, and Treo) and new ways to communicate (e.g blogs, Facebook, and Twitter). Here, we suggest that the richness of communication media not only depends on the communication media, but also on device used and the technological surroundings in which communication takes place.

On the other hand, little empirical evidence exists on the relation between perceived richness of a medium or communication channel and its choice. Some theories, like media synchrony theory (Dennis, Fuller, \& Valacich, 2008), present theoretical models in which the choice of communication media is dependent on the relation between the perceived richness of the medium and the type of communication process undertaken (e.g conveyance and convergence). However, it is still necessary to verify these relations through empirical studies, and it is here that the third important line of investigation on the richness of channels is suggested. Due to their nature and to the results obtained in this investigation, these would have to be undertaken 
in experimental form and longitudinally in order to be able to take in the time factor and to introduce extraneous variables that could better explain the results. The complexity of these studies is high due to the great number of variables and aspects to take into account, and to the large amount of differences that exist between individuals and media when communicating.

Finally, a revision of the literature shows the small number of investigations that have examined the effects of diverse communication media (based on their richness) on individuals such as satisfaction with the communication, satisfaction with the communication result, the perceived quality of communication, and the perceived quality of the communication result. Although Byrne and LeMay (2006) undertook a first analysis of some of these effects, their investigation did not take into account neither knowledgebuilding experiences proposed by Carlson and Zmud (1999) nor the time factor because of its static cross-sectional research design. The inclusion of these elements in the investigation could improve the understanding of the decisions made by an organization's managers and employees when communicating.

\section{References}

Barry, B., \& Smithey Fulmer, I. (2004). The medium and the message: The adaptive use of communication media in dyadic influence. Academy of Management Review, 29(2), 272-292.

Boyd, B., Gove, S., \& Hitt, M. (2005). Construct measurement in strategic management research: illusion or reality? Strategic Management Journal 26(3), 239-257.

Byrne, Z., \& LeMay, E. (2006). Different media for organizational communication: Perceptions of quality and satisfaction. Journal of Business and Psychology, 21(2), 149-173. 
Carlson, J., \& Zmud, R. (1999). Channel expansion theory and the experimental nature of media richness perceptions. Academy of Management Journal, 42(2), 153-170.

Collins, D. (2003). Pretesting survey instruments: An overview of cognitive methods. Quality of Life Research, 12(3), 229-238.

D'Urso, S. C., \& Rains, S. A. (2008). Examining the scope of channel expansion - A test of channel expansion theory with new and traditional communication media. Management Communication Quarterly, 21(4), 486-507.

Daft, R., \& Lengel, R. (1984). Information richness: A new approach to managerial behavior and organizaton design. Research in Organizational Behavior, 6, 191-233.

Daft, R., \& Lengel, R. (1986). Organizational information requirements, media richness and structural design. Management Science, 32(5), 554571.

Dahle, T. (1954). An objective and comparative study of five methods of transmitting information to business and industrial employees. Speech Monographs, 21, 21-28.

Dennis, A., Fuller, R., \& Valacich, J. (2008). Media, Tasks, and Communication Processes: A Theory of Media Synchronicity. MIS Quarterly, 32(3), 575-600.

Dennis, A., \& Kinney, S. (1998). Testing media richness theory in the new media: The effects of cues, feedbacks, and task equivocality. Information System Research, 9(3), 256-274.

Dennis, A., \& Valacich, J. (1999). Rethinking Media Richness: Towards a Theory of Media Synchronicity. Paper presented at the 32th Hawaii International Conference on system Sciences, Los Alamitos, CA.

Drennan, J. (2003). Cognitive interviewing: verbal data in the design and pretesting of questionnaires. Journal of Advanced Nursing, 42(1), 5763. 
Ferry, D., Kydd, C., \& Sawyer, J. (2001). Measuring facts of media richness. The Journal of Computer Information Systems, 41(4), 69-78.

Fulk, J. (1993). Social construction of communication technology. Academy of Management Journal, 36(5), 921-950.

Fulk, J., \& Boyd, B. (1991). Emerging theories of communication in organizations. Journal of Management, 17(2), 407-446.

Hasty, B., Massey, A., \& Brown, S. (2006). Role-based experiences, media perceptions, and knowledge transfer success in virtual dyads. Group Decision and Negotiation, 15, 367-387.

Hitt, M., Gimeno, J., \& Hoskisson, R. (1998). Current and future research methods in strategic management. Organizational Research Methods, $1,6-44$.

Kishi, M. (2008). Perceptions and use of electronic media: Testing the relationship between organizational interpretation differences and media richness. Information \& Management, 45(5), 281-287.

Lewicki, R., Barry, B., Saunders, D., \& Minton, J. (2003). Negotiation (4th ed.). Boston: McGraw-Hill/Irwin.

Markus, M. (1987). Toward a critical mass theory of interactive media Universal access, interdependence and diffusion. Communication Research, 14(5), 491-511.

Nunnally, J. C., \& Bernstein, I. H. (1994). Psychometric Theory (3rd Ed ed.). New York: McGraw-Hill.

Ravenscroft, A., \& Matheson, M. (2001). Carpe diem: Models and methodologies for designing engaging and interactive e-learning discourse. Paper presented at the Proceedings IEEE international conference on advanced learning technologies Los Alamitos, CA.

Rice, R. (1992). Task analyzability, use of new media, and effectiveness: A multi-site exploration of media richness. Organization Science, 3(4), 475-500. 
Rice, R., \& Love, G. (1987). Electronic emotion. Communication Research, $14,85-108$.

Rothaermel, F. T., Hitt, M. A., \& Jobe, L. A. (2006). Balancing vertical integration and strategic outsourcing: Effects on product portfolio, product success, and firm performance. Strategic Management Journal, 27(11), 1033-1056.

Schmitz, J., \& Fulk, J. (1991). Organizational colleagues, media richness, and electronic mail - a test of the social-influence model of technology use. Communication Research, 18(4), 487-523.

Short, J., Williams, E., \& Christie, B. (1976). The social psychology of telecommunications. London: John Wiley \& Sons.

Te'eni, D., Sagie, A., Schwartz, D., Zaidman, N., \& Amichai-Hamburger, Y. (2001). The process of organizational communication: A model and field study. IEEE Transactions on Professional Communication, 44(1), 6-20.

Timmerman, C. E. (2002). The moderating effect of mindlessness/mindfulness upon media richness and social influence explanations of organizational media use. Communication Monographs, 69(2), 111132.

Timmerman, C. E., \& Madhavapeddi, S. N. (2008). Perceptions of organizational media richness: Channel expansion effects for electronic and traditional media across richness dimensions. IEEE Transactions on Professional Communication, 51(1), 18-32.

Trevino, L., Lengel, R., \& Daft, R. (1987). Media symbolism, media richness, and media choice in organizations. Communication Research, 14(5), 553-574.

Vishwanath, A. (2006). The effect of the number of opinion seekers and leaders on technology attitudes and choices. Human Communication Research, 32(3), 322-350. 
Walther, J. (1992). Interpersonal effects in computer-mediated interaction: A relational perspective. Communication Research, 19, 52-90.

Walther, J. (1995). Relational aspects of computer-mediated communication experimental-observations over time. Organization Science, 6(2), 186203.

Williamson, J., Ranyard, R., \& Cuthbert, L. (2000). A conversation-based process tracing method for use with naturalistic decisions: An evaluation study. British Journal of Psychology, 91, 203-221.

Yates, J., \& Orlikowski, W. (1992). Genres of organizational communication A structurational approach to studying communication and media. Academy of Management Review, 17(2), 299-326. 


\section{$\underline{\text { Appendix (Scales) }}$}

\section{Perceived Media Richness}

1. The online discussion forum feature allows us to give and receive timely feedback.

2. The online discussion forum feature allows us to tailor our messages to our own personal requirements.

3. The online discussion forum feature allows us to communicate a variety of different cues (such as emotional tone, attitude, or formality) in our messages.

4. The online discussion forum feature allows us use rich and varied language in our messages.

5. I could easily explain things using the online discussion forum feature.

6. The online discussion forum feature helped us communicate quickly.

7. The online discussion forum feature helped us to better understand each other.

\section{Experience with the online discussion forum}

1. I am very experienced using the online discussion forum feature.

2. I feel that the online discussion forum feature is easy to use. 
3. I feel competent using the online discussion forum feature.

4. I understand how to use all of the options of the online discussion forum feature.

5. I feel comfortable using the online discussion forum feature.

6. I feel that I am a novice using the online discussion forum feature.

\section{Experience with Messaging Topic}

1. I feel that I am experienced with the course contents.

2. I feel that I am well versed in the concepts associated with the course contents.

3. I do not feel knowledgeable about the course contents.

\section{Experience with Communication Partner}

1. Overall, I feel that I know my classmates and lecturers well.

2. I feel comfortable communicating emotional issues with my classmates and lecturers.

3. I feel comfortable discussing personal or private issues with my classmates and lecturers.

4. I feel close to my classmates and lecturers.

5. I feel that I am not familiar with my classmates and lecturers. 
6. I feel involved with my classmates and lecturers.

\section{Surveillance}

1. The online discussion forum could be control and surveillance easily.

2. The information in the online discussion forum could be gathered and utilized easily by other people. 


\section{TABLES AND FIGURES}

Table 1

Descriptive Statistics and Correlation

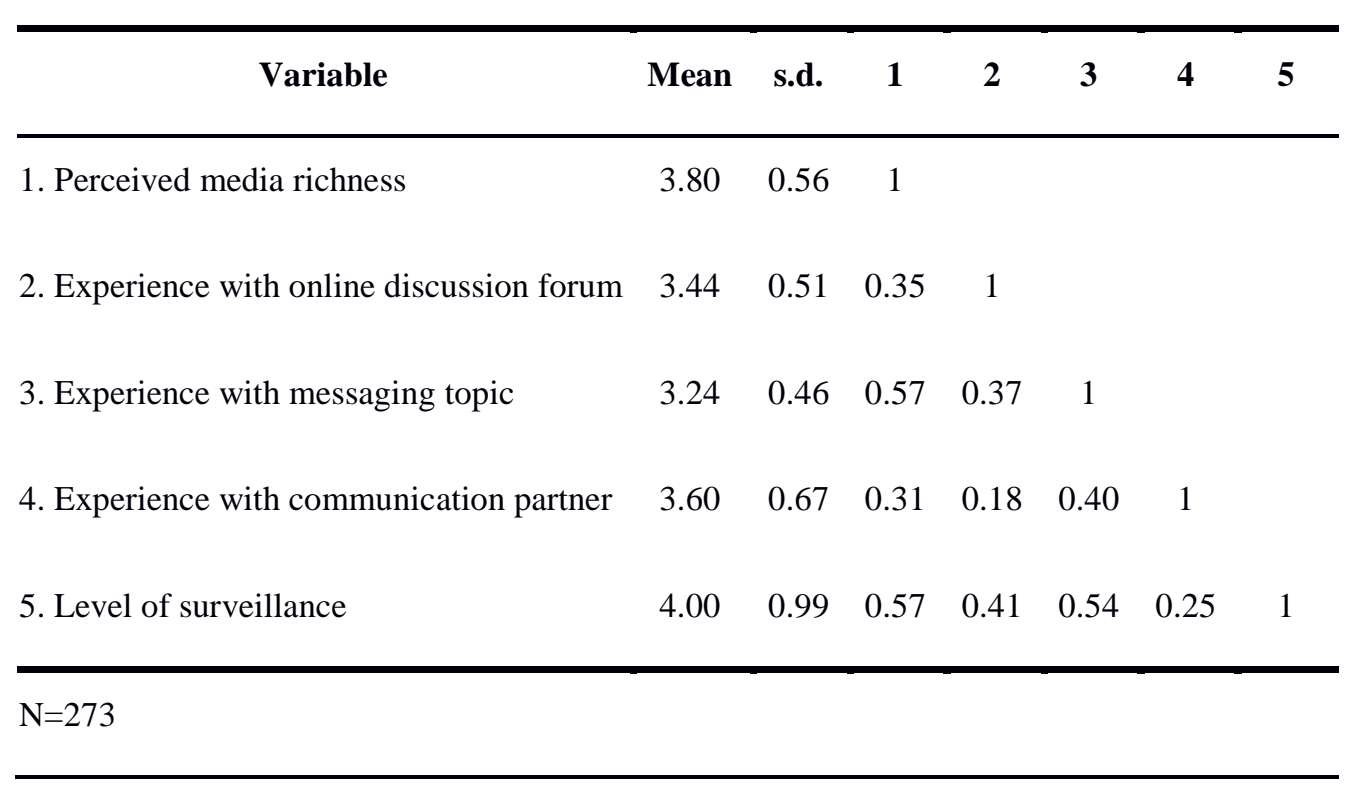


Table 2

Results of Regression Analysis

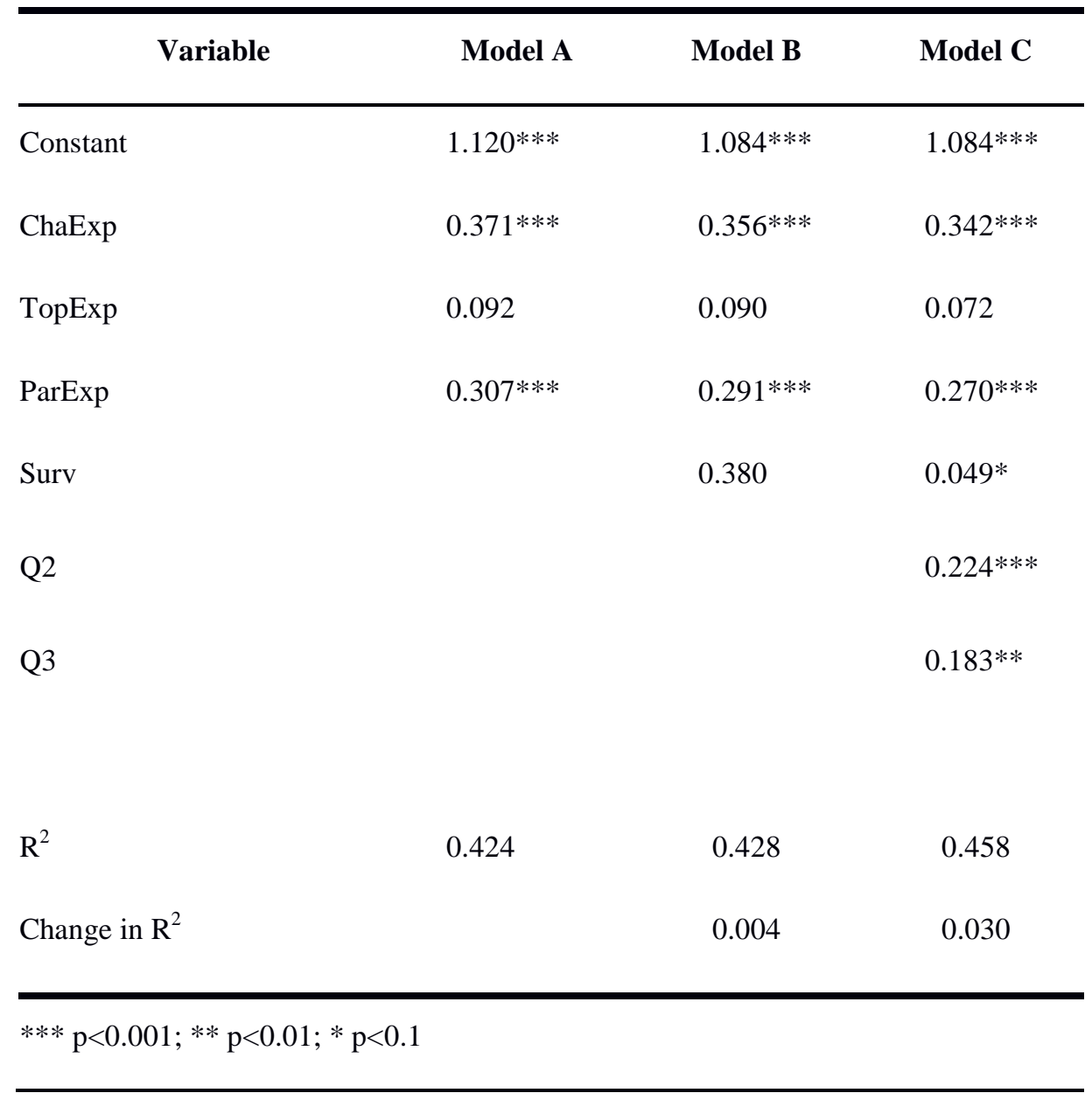

